\title{
The importance of submalleolar deformity in determining leg length discrepancy
}

\author{
Ayman Ali ${ }^{a, *}$, Michael Walsh ${ }^{a}$, Timothy O'Brien ${ }^{a}$, Borislav D. Dimitrov ${ }^{b, c}$ \\ ${ }^{a}$ Central Remedial Clinic, Vernon Avenue, Clontarf, Dublin 3, Ireland \\ ${ }^{\mathrm{b}}$ Department of General Practice, Royal College of Surgeons in Ireland, 123 St. Stephens Green, Dublin 2, Ireland \\ ${ }^{c}$ Academic Unit of Primary Care and Population Sciences, University of Southampton, Southampton SO16 6YD, \\ United Kingdom
}

\section{A R T I C L E I N F O}

\section{Article history:}

Received 2 October 2013

Received in revised form

10 December 2013

Accepted 12 December 2013

Available online 8 January 2014

Keywords:

Leg length discrepancy

Measurements

3-D gait analysis

Foot position

\begin{abstract}
A B S T R A C T
Background and purpose: The association of leg length discrepancy (LLD) with a number of clinical disorders has made its determination a significant part of the physical examination. We believe that submalleolar causes of LLD may be under-acknowledged. The most common clinical method used to measure LLD is by tape from the anterior superior iliac spine (ASIS) to medial malleolus which disregards the potential for LLD arising from asymmetry in the foot distal to the tibiotalar joint.

Methods: The present pilot study involves a group of 5 volunteers (experimental group) and a group of 3 patients with flexible flat feet (clinical study). The differences in tibial tubercle height from the ground between full pronation and full supination were measured using the CODA MPX $30^{\circledR}$ system (Charnwood Dynamics Limited, Leicestershire, England). Correlations of the patterns within each group were produced.

Results: A significant relationship with leg lengths was found in the experimental group when they induced maximum pronation ( $R$-squared $=0.62, p=0.007$ ) while an inverse relationship occurred with supination, although marginally significant $(R$-squared $=0.37$, $p=0.064)$.

Conclusions: We have demonstrated that significant leg length discrepancy can occur in patients who do not have obvious deformity when non weight bearing. We recommend using the blocks method routinely. Appropriately measuring LLD is of vital importance to properly diagnosing and treating patients with unequal leg lengths or related symptoms.

(c) 2013 Royal College of Surgeons of Edinburgh (Scottish charity number SC005317) and

Royal College of Surgeons in Ireland. Published by Elsevier Ltd. All rights reserved.
\end{abstract}

\section{Introduction}

Leg length discrepancy (LLD), defined as the difference in lengths of the two lower limbs, is very common, occurring in up to $70 \%$ of the population. ${ }^{1}$ The diagnosis of LLD is usually made on physical examination and is confirmed and measured with imaging studies. ${ }^{2}$ LLD can be subdivided into two etiological groups: a structural LLD (SLLD) defined as those associated with a shortening of bony structures, and a functional LLD (FLLD) defined as those that result from altered

\footnotetext{
* Corresponding author. Tel.: +353 872121876; fax: +353 18336633.

E-mail addresses: drayman891@hotmail.com (A. Ali), mwalsh@crc.ie (M. Walsh), proftobrien@hotmail.com (T. O’Brien), b.dimitrov@ soton.ac.uk, borislav.d.dimitrov@gmail.com (B.D. Dimitrov).

1479-666X/\$ - see front matter ( 2013 Royal College of Surgeons of Edinburgh (Scottish charity number SC005317) and Royal College of Surgeons in Ireland. Published by Elsevier Ltd. All rights reserved.

http://dx.doi.org/10.1016/j.surge.2013.12.003
} 
mechanics of the lower extremities. ${ }^{3}$ SLLD, also known as true LLD, may be congenital or acquired. Common congenital causes include dislocation of the hip and hemiatrophy or hemihypertrophy with skeletal involvement. Acquired causes can be due to infections, paralysis, tumours, surgical procedures like prosthetic hip replacement and mechanical causes such as slipped capital femoral epiphysis. Functional, or apparent LLD, can result from muscle tightness or weakness and joint contractures. Other causes relating to joint position include hip abduction/adduction, knee hyperextension due to quadriceps and hamstring weakness, knee flexion contracture, equinus deformity and pronation or supination of one foot relative to the other. ${ }^{4}$

According to the literature the commonly used clinical method to measure LLD is the tape measurement from anterior superior iliac spine (ASIS) to lateral malleolus, ${ }^{1}$ and (ASIS) to medial malleolus. ${ }^{5}$ Measuring to only the malleoli disregards the potential for LLD arising from asymmetry in the foot distal to the tibiotalar joint (e.g., calcaneal fracture, developmental abnormalities, degenerative arthritis, Charcot foot, collapsed medial longitudinal arch). ${ }^{6}$ The authors of this study believe that the role of submalleolar deformity and its effect on functional length of the lower limb is under-appreciated in clinical practice. The authors have reported previously on the effects of pelvic, hip, knee and ankle joint kinematics on limb length. ${ }^{7}$ However, there is a scarcity of knowledge in relation to the magnitude of effect on limb height of clinical changes in submalleolar joint mechanics.

The purpose of this pilot study was two-fold. We aimed to: 1) measure the experimental effect of pronation and supination position of the foot on lower limb length in healthy subjects; and 2) confirm the experimental findings in a clinical population.

\section{Methods and materials}

\section{Experimental study}

Five volunteer senior physiotherapists, experienced in therapeutic movement and with no foot deformities, were recruited. The senior physiotherapists were chosen as study subjects because they would have understood the command of moving from full pronation to full supination without significant alteration of body posture and because of their experience to control their own subject posture. Full 3-D lower limb kinematic evaluation was carried out using the CODA MPX $30^{\circledR}$ motion analysis system (Charnwood Dynamics Limited, Leicestershire, England). This is a three-dimensional pre-calibrated system which captures infra-red light signals from markers placed on anatomical landmarks in accordance with the model outlined by the system manufacturer, the model is equivalent to the standard model used during lower body gait analysis. ${ }^{8,9}$ The hip joint centres are derived relative to the pelvic segment based on the model of Bell et al. ${ }^{10}$ For each subject the CODA took 100 observations as the foot moved from supination to neutral to pronation and the measurement value was extracted from these observations. In addition to the standard gait model markers were also placed on the tibial tubercles to allow measurement of the height of a fixed bony point on the lower limb from the ground as the submalleolar deformity is more evident on weight bearing. The tibial tuberosity is situated on the bone just proximal to the joints being considered in this study; the ankle and subtalar joints. The marker was not per se measuring leg length, but the change in height of the marker from the floor surface, which is in fact the same as the change in leg length, in the positions of supination and pronation.

Subjects were asked to stand in a comfortable relaxed position with feet a shoulder width apart. Data was captured at rest. Subjects were then asked to move one foot at a time into positions of full pronation and full supination without altering other joint positions. The process was repeated for the contralateral foot. Prior to formal data collection subjects were given a period of time to practice the movements. Three files were captured for each foot and data averaged from these files. Kinematic data for pronation and supination of the feet and corresponding height of tibial tubercle from the floor were retrieved for analysis.

\section{Clinical study}

A small convenience sample of three patients with clinically diagnosed correctable submalleolar deformities secondary to flexible flat foot were recruited to the study for the purposes of clinical validation of the experimental findings. All subjects presented with significant pronation deformities (two subjects bilaterally, one subject unilaterally). The same marker placement protocol as for the experimental normal group was used. On this occasion testing differed in that data was captured firstly with the patients in their natural weightbearing foot positions of excessive pronation. A second data set was captured while the foot was corrected manually to a
A)

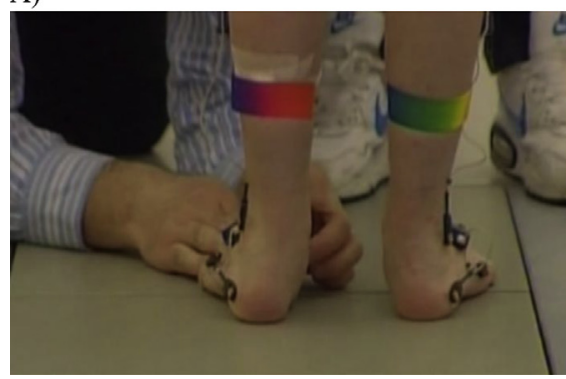

B)

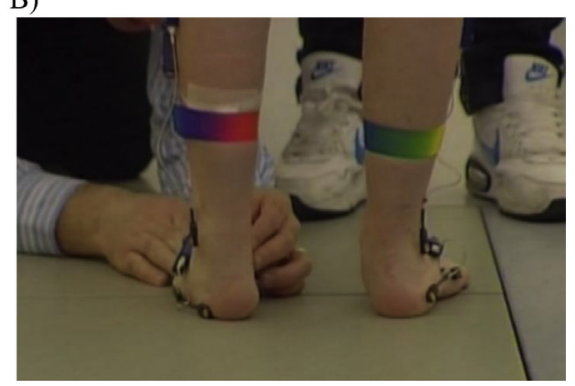

Fig. 1 - Foot position before and after correction of the deformity. A) Uncorrected; B) Corrected. 
subtalar neutral position by an experienced therapist (Fig. 1). These two datasets were evaluated to assess the effect on the height of the limb from the ground of correcting the submalleolar deformity.

There was only one assessor, a very experienced gait laboratory therapist and the participants served as their own controls. The markers were only applied once and not changed thereafter during the experimental movement sequence. Once the marker set was applied it was not disturbed after that. The study subjects went through a sequence of movement from pronation to supination. The comparison was height of the tibial tubercle between these two positions in the same subject with the same marker set. The study protocol was approved by the Research Ethics Committee of the Central Remedial Clinic, Dublin, Ireland.

\section{Statistical analysis}

As a proof-of-concept study, the pilot design did neither require larger sample nor power calculation or sample size calculation a priori. Descriptive and exploratory statistical analyses were applied to the experimental and clinical data. Data are presented as mean $( \pm \mathrm{SD})$ and frequency (percentage), as appropriate. The variables that were not normally distributed were analysed by non-parametric methods and tests. A regression analysis with calculation of correlation coefficient $(R)$ and coefficient of determination $\left(R^{2}\right)$ was performed on foot position in the coronal plane versus height of the tibial tubercle from the ground within each group of the study subjects. Statistical significance was assumed at $p<0.05$, unless stated otherwise. All analyses were done with STATA/IC 11.2 software.

\section{Results}

\section{Experimental study}

We found that the movement of the tibial tubercle marker as the foot moves from pronation to supination was associated

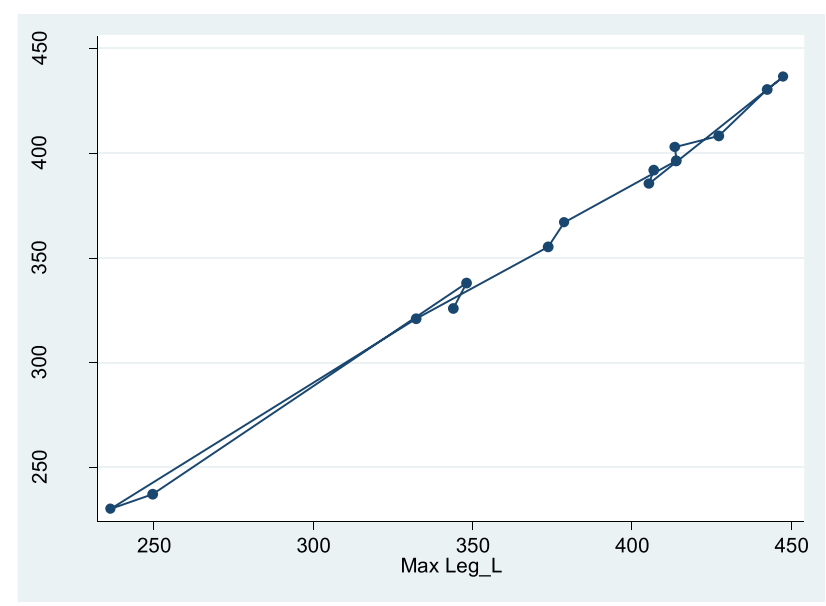

Fig. 2 - Movement of the tibial tubercle marker as the foot moves from pronation (Min Leg L) to supination (Max Leg L) with overall increase in leg length. with overall increase in leg length (Fig. 2). In this group we found important relationships of leg length with maximum pronation $\left(R^{2}=0.62, p=0.007\right)$ and maximum supination $\left(R^{2}=0.37, p=0.064\right)$ (Table 1$)$.

It can be clearly seen on Fig. 2 that the height of the tibial tubercle from the ground increases as the foot is moved from pronation to supination. In the experimental group (10 feet) the mean change in the foot position was from $-10.8^{\circ}$ to $15.9^{\circ}$ (standard deviation 4.23 and 3.75 , range 18.1 to -4.9 and 9.6-22.9, respectively). This resulted in a corresponding mean change in the height of the tibial tubercle from the ground of $\sim 1 \mathrm{~cm}$ (mean 399.9 to $410.5 \mathrm{~mm}$, standard deviation 24.38 and 24.014 , range $363.8-437.2$ and $373.8-447.5$, respectively).

\section{Clinical group}

In the clinical group there were similar findings to the experimental group. As each foot was moved from a pathological pronated position to a subtalar neutral position there was a corresponding increase in the height of the tibial tubercle marker from the floor of the ipsilateral limb (Fig. 3). There was a weak but significant correlation between the height of the tibial tubercle from the floor and changes in foot position (Kendall's tau $=0.200, p=0.62$ ) (Table 1 ).

The mean change in foot position in this group was from $-11.4^{\circ}$ to $8.2^{\circ}$ (standard deviation 6.72 and 5.67, range -15.37-0.54 and 2.88-17.41, respectively). The corresponding mean change in height of the tibial tubercle from the floor was of the order from 291.3 to $298.9 \mathrm{~mm}$ (standard deviation 51.6 and 53.3, range 231.9-338.4 and 234.4-347.9, respectively).

In the subject with unilateral valgus deformity the mean limb length in a resting position was $321.4 \mathrm{~mm}$, when the foot deformity was corrected it changed to $332.3 \mathrm{~mm}$. This provides very clear evidence for clinical validation of the experimental findings.

\section{Discussion}

Leg length discrepancy as a cause of symptoms has been widely discussed in the literature. ${ }^{1-4}$ The magnitude of discrepancy that causes symptoms is also a cause of debate. It is generally agreed that a discrepancy of $\geq 1 \mathrm{~cm}$ is clinically significant. ${ }^{11}$ Treatment of LLD ranges from shoe inserts to various surgical techniques including limb lengthening and shortening, and epiphysiodesis. There is disagreement

\begin{tabular}{lcc}
\hline Table $\mathbf{1}$ - Correlation analysis of leg lengths. & \\
\hline Variable & $\begin{array}{c}\text { Correlation } \\
\text { coefficient } R\end{array}$ & $p$-Value \\
\hline $\begin{array}{l}\text { Maximum pronation } \\
(n=10 \text { legs }) \text { - experimental }\end{array}$ & 0.79 & 0.007 \\
$\begin{array}{l}\text { Maximum supination } \\
(n=10 \text { legs }) \text { - experimental }\end{array}$ & -0.61 & 0.064 \\
$\begin{array}{l}\text { Maximum pronation } \\
(n=5 \text { legs }) \text { - clinical }\end{array}$ & $0.20^{\mathrm{a}}$ & 0.624 \\
\hline a & & \\
\hline
\end{tabular}




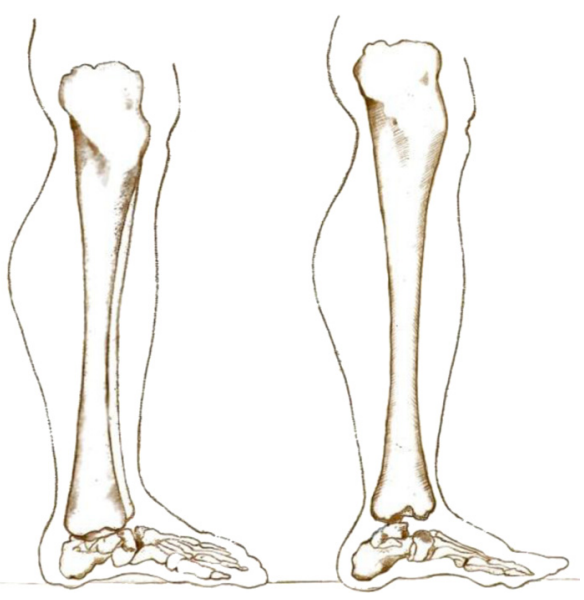

Fig. 3 - Diagrammatic representation of the change in length of the leg when changing from pronation (left) to supination (right).

regarding the correct treatment in regards to magnitude of LLD. Reid and Smith suggest dividing LLD into three categories, mild (0-30 mm), moderate $(30-60 \mathrm{~mm})$, and severe (>60 mm), where mild cases should either go untreated or treated non-surgically, moderate cases should be dealt with on a case by case basis, and severe cases should be corrected surgically. ${ }^{12}$

The contribution to leg length discrepancy at different anatomical levels has been previously reported. ${ }^{7}$ The most common clinical tool used to measure LLD is the traditional measuring tape using the anatomical reference points from ASIS to medial malleolus. However, this ignores the effects of deformity below the level of the ankle joint. We believe that the use of blocks in standing is a more comprehensive clinical measure and accounts for weight bearing changes below the level of the ankle. Likewise, clinical checking of the correctability of submalleolar deformity and the effect of the corrected position on symmetry of limb lengths is equally an important consideration.

Our experimental study in a normal group demonstrated that changes in foot position from maximum pronation to supination resulted in a change in limb length of $1 \mathrm{~cm}$. Likewise, in our clinical group we demonstrated very clearly that correction of a pathological pronated foot can result in a change of limb height of $\sim 1 \mathrm{~cm}$. We believe that this represents an important clinical consideration when deciding on how to manage LLD in a clinical population who present with foot deformities and LLD problems such as in cerebral palsy hemiplegia where there is already significant shortening of the affected lower limb. For example if a hemiplegic patient has a measured limb shortening to the medial malleolus of one centimetre, the presence of another centimetre shortening due to a pronated foot might be sufficient to decide on surgical correction.

We have demonstrated that mobile changes of the foot from pronation to supination results in a change in limb length of about one centimetre. Where a patient has a limb length discrepancy that is considered borderline for surgical intervention, the presence or absence of a foot deformity might be the decisive factor. We conclude that any investigation of limb length discrepancy, both clinical and radiological, include consideration of submalleolar structures and their functional weight bearing position.

\section{Conclusions}

Finally the importance of this study is that we have demonstrated that significant leg length discrepancy can occur in patients who do not have obvious deformity when non weight bearing. The use of blocks under the short leg to level the pelvis has been shown to be the most reliable clinical test for LLD. ${ }^{1}$ Clinical measurements are less costly, are more readily available, and are not associated with the risks related to exposure to radiation. ${ }^{13}$ Further confirmation of our findings is needed; however, a recommendation for the use of blocks routinely is warranted as this method takes into account the disparity in foot height between the two limbs. Clearly, it also helps determining the functional LLD (which may be different from the actual LLD) by using varying heights of the block to establish the additional length required for the patient to feel level. ${ }^{14}$ We may also suggest that the effects of a mobile submalleolar deformity be accounted for in any such assessment.

In patients with unilateral cerebral palsy who already may have shown a significant leg length discrepancy ${ }^{15}$ the additional submalleolar deformity adds to the leg length discrepancy and should be taken into account by correcting the deformity or if it is not correctable then by incorporating a raise. The use of appropriate clinical methods and imaging modalities for measuring the LLD is vital to properly treat a patient with unequal leg lengths or related symptoms ${ }^{14}$

\section{R E F E R E N C E S}

1. Woerman AL, Binder-Macleod SA. Leg length discrepancy assessment: accuracv and precision in five clinical methods of evaluation*. J Orthop Sports Phys Ther 1984;5(5):230-9.

2. Sabharwal S, Zhao C, McKeon JJ, McClemens E, Edgar M, Behrens F. Computed radiographic measurement of limblength discrepancy. Full-length standing anteroposterior radiograph compared with scanogram. J Bone Joint Surg Am 2006 Oct;88(10):2243-51.

3. Blake RL, Ferguson H. Limb length discrepancies. J Am Podiatr Med Assoc 1992 Jan;82(1):33-8.

4. Gurney B. Leg length discrepancy. Gait Posture 2002 Apr;15(2):195-206.

5. Beattie P, Isaacson K, Riddle DL, Rothstein JM. Validity of derived measurements of leg-length differences obtained by use of a tape measure. Phys Ther 1990 Mar;70(3):150-7.

6. Hanada E, Kirby RL, Mitchell M, Swuste JM. Measuring leglength discrepancy by the "iliac crest palpation and book correction" method: reliability and validity. Arch Phys Med Rehabil 2001 Jul;82(7):938-42.

7. Walsh M, Connolly P, Jenkinson A, O’Brien T. Leg length discrepancy-an experimental study of compensatory changes in three dimensions using gait analysis. Gait Posture 2000 Oct;12(2):156-61. 
8. Davis III RB, Ounpuu S, Tyburski D, Gage JR. A gait analysis data collection and reduction technique. Hum Mou Sci 1991;10:575-87.

9. Kadaba MP, Ramakrishnan HK, Wootten ME. Measurement of lower extremity kinematics during level walking. J Orthop Res 1990 May;8(3):383-92.

10. Bell AL, Pedersen DR, Brand RA. A comparison of the accuracy of several hip center location prediction methods. J Biomech 1990;23(6):617-21.

11. Harvey WF, Yang M, Cooke TD, Segal NA, Lane N, Lewis CE, et al. Association of leg-length inequality with knee osteoarthritis: a cohort study. Ann Intern Med 2010 Mar 2;152(5):287-95.
12. Reid DC, Smith B. Leg length inequality: a review of etiology and management. Physiother Can 1984;36:177-82.

13. Paley D, Bhave A, Herzenberg JE, Bowen JR. Multiplier method for predicting limb-length discrepancy. J Bone Joint Surg Am 2000 Oct;82-A(10):1432-46.

14. Sabharwal S, Kumar A. Methods for assessing leg length discrepancy. Clin Orthop Relat Res. 2008 Dec;466(12):2910-22.

15. McKenna J, Walsh M, Jenkinson A, Hewart P, O’Brien T. Leg length discrepancy and equinus deformity in the hemiplegic cerebral palsy patient. In: Irish Orthopaedic Association/ Welsh Orthopaedic Society Meeting. J Bone Joint Surg Br 2002;84-B(Supp I):1-a-1 [Abstract]. 